\title{
Verleihung der Salomon-Neumann-Medaille 2011
}

Ilona Kickbusch

\section{Bibliografie}

DOI http://dx.doi.org/ 10.1055/s-0032-1311557

Gesundheitswesen 2012;

74: $219-220$

(c) Georg Thieme Verlag KG

Stuttgart · New York

ISSN 0941-3790

Korrespondenzadresse

Prof. llona Kickbusch Kickbusch Health Consult http://www.ilonakickbusch. com

info@ilonakickbusch.com
Sehr geehrte Frau Senatorin, liebe Preisträgerinnen, liebe Kolleginnen und Kollegen -

Ulrike Maschwesky-Schneider steht exemplarisch für die Grundsätze und Werte dieser Gesellschaft - der DGSMP. Sie vermittelt sie in Lehre und Forschung, in der Verbindung zur Praxis sowie in Ämtern und Funktionen. Der Bundesverband der Frauengesundheitszentren steht für die gesundheitsbezogene Anwaltschaft, die es weiterhin für Frauen und ihre Gesundheit in dieser Gesellschaft braucht - aber auch für sehr notwendige konkrete Unterstützung vor Ort. Es ist mir eine außerordentliche Ehre für sie die Laudatio halten zu dürfen. Ich hoffe ich werde beiden gerecht.

Es beginnt mit dem Körper. „Der individuelle Körper muss sowohl als das unmittelbarste Terrain betrachtet werden, wo soziale Wahrheiten und soziale Widersprüche ausgetragen werden, als auch als der Ort von persönlichem und sozialem Widerstand, Kampf und Kreativität." so Nancy ScheperHughes, eine radikale Anthropologin, im Jahr 1987. Diese Worte würden von dem auf seine Weise radikalen Namensgeber dieses Preises sehr gut verstanden werden. Den Preisträgerinnen sind sie Programm.

Den Aktivistinnen der Frauengesundheitsbewegung war genau diese doppelte Bedeutung des Körpers stets Thema - am besten ausgedrückt durch die Arbeit des Boston Women's Health Collective und dem Erscheinen 1970 von „Our Bodies - Ourselves“ dem ersten umfassenden Gesundheitshandbuch für Frauen - zuerst in den USA dann in immer mehr Ländern, inzwischen auch in vielen Entwicklungsländern. Die deutschen Frauengesundheitszentren haben bis heute gegen viel Widerstand und mit wenig Geld die hierin verankerten Grundsätze der Selbstbestimmung umgesetzt - für Frauen aller Altersstufen und unterschiedlicher Herkunft.

Die Entdeckung der speziellen Dynamik zwischen dem individuellen Körper, dem persönlichen Empowerment und von sozialer Lebenswelt, Veränderung und Bewegung ist bis heute Thema der Frauengesundheit. Und sie ist darüber hinaus zum Thema einer modernen Auffassung des Gesundheitshandelns geworden - wie in der Ottawa Charter 1986 dargelegt. Vertreterinnen des Boston Women's Health Collective waren auf der historischen Tagung, die sich in diesem November 2011 zum 25sten mal jährt, ebenfalls anwesend. Die Erkenntnisse der Frauengesundheitsbewegung haben somit weit in die Ansätze des Public health hineingewirkt. Darum ging es ja auch: eine neue öffentliche Gesundheit//a new public health. Selbstbestimmt - partizipativ - Lebenswelt bezogen.

Die ersten Frauengesundheitszentren in Deutschland nannten sich selbstbewusst feministisch das stand für den anderen Blick und für die Parteinahme für die Rechte und Interessen von Frauen, für sexuelle und reproduktive Selbstbestimmung und für eine grundlegende Umwälzung der Geschlechter- und Sexualbeziehungen. So einfach - bis heute. „Von der gesellschaftlichen Ungleichheit zwischen Mann und Frau ausgehend, zielt der Feminismus auf eine verbesserte Lage der Frau und ihre faktische Gleichstellung in der Gesellschaft". Heute vermeiden wir das Wort gerne und sprechen dezenter vom SDH Ansatz: den sozialen Determinanten von Gesundheit.

Der feministischen Praxis der Frauengesundheitszentren folgte die feministische Wissenschaft. In der Frauengesundheitsbewegung und der Frauengesundheitsforschung manifestierte sich eine historisch neue Form der „Medizin als soziale Wissenschaft“. Der Blick auf „das andere Geschlecht" eröffnete neue Dimensionen der Ungleichheit und konfrontierte den Forschungsbetrieb mit unangenehmen Wahrheiten und komplexeren Forschungsfragen. Das galt nicht nur für die Medizin, die sich stets als objektiv und neutral verstanden hatte, sondern auch für die sozial-medizinische und gesundheits- und medizinsoziologische Forschung, die sich als objektiv auf der Suche nach dem „sozialen“ verstanden hatte. Sie mussten feststellen, dass ihr Blick 2-fach eingeschränkt war: zum einen erfolgte eine Normierung des Körpers durch den medizinischen Blick zum anderen verengte sich die soziale Realität durch den männlichen Blick. Beigelegt sind diese grundlegenden Konfliktlinien bis heute nicht im politischen Raum werden sie stellvertreterhaft ausgefochten zwischen Frauen wie Schwarzer, Roche und Schröder, im sozialen Raum des Alltags wird der Frauenkörper ästhetisch-pornografisch plakatiert und ansonsten werden mithilfe des Gender Mainstreaming die Konflikte domestiziert. Fein wird zwischen dem sozialem Geschlecht (Gender) und biologischem Geschlecht (Sex) unterschieden und wir werden damit vor jeder Radikalität geschützt. Männergesundheit, viel weniger konfliktträchtig und ein neuer Wachstumsmarkt, hat Konjunktur. „Gender medicine“ hat fast jeden Bezug zur „sozialen Wissenschaft" verloren. Der Körper als Text sozialer Ungleichheit - die Kultur ist auf den Körper geschrieben - wird nicht mehr erfasst. Das gilt auch für den schleichenden Zugriff auf den Männerkörper. 
Nun sollte dies ja kein historischer Exkurs zum Feminismus werden - sondern eine Laudatio auf Ulrike Maschewsky-Schneider und auf die Frauengesundheitszentren. Wie aber könnte man außerordentliche Leistung würdigen, wenn man sie nicht in den sozialen und politischen Kontext stellen würde? Ist nicht schon die Tatsache, dass es 25 Preisträger der Salomon Neumann Medaille braucht bis man es auf 2 Frauen (und zugegeben 16 Frauengesundheitszentren) bringt ein Ausdruck des noch nicht erreichten? Ist es nicht auch Hinweis auf die Schwierigkeiten, die es galt zu überwinden?

Man schaue sich an wie konsequent die Frauengesundheitszentren ihren Auftrag erfüllt haben. Man schaue sich an wie konsequent Ulrike Maschewsky-Schneider die „Medizin als soziale Wissenschaft" zu ihrem Lebensthema gemacht hat. Sie hat sich den Themen zugewandt, die von höchster Public Health Relevanz sind, aber im gesundheitspolitischen Handeln und in der universitäts-politischen Verankerung stets den kürzeren ziehen: Prävention, Gesundheitsförderung, Public Health, Frauengesundheit, Gesundheit sozial benachteiligter Bevölkerungsgruppen, Gemeindeinterventionen, Rauchen, Gewalt. Sie stellt die zentrale Frage: was macht Frauen krank? Sie untersucht den gender bias in der Gesundheitsforschung. Sie erforscht die sozialen Ursachen des Herzinfarkts. Sie zeigt auf wie die Berücksichtigung sozialer Faktoren zu erfolgreicher Prävention führen kann und ist aktiv in der Umsetzung in der „Bremer Arbeitsstelle für Gesundheitsförderung am BIPS“. Sie engagiert sich für eine transparente - auch frauenspezifische - Gesundheitsberichterstattung auf lokaler (hier in Bremen) und auf nationaler Ebene. Sie versucht stets handlungsrelevant zu sein - den Bezug zur Praxis klar aufrecht zu erhalten.

In all diesen Themen liegt die soziale Sprengkraft der Verhältnisse - für die Praxis und die Wissenschaft. Ulrike MaschewskySchneider hat sie - auch hier eng in der „statistischen“ Tradition von Salomon Neumann - mit harten „objektiven“ Daten belegt und gleichzeitig auf qualitativen Studien bestanden. Es gilt sowohl die objektive wie die subjektive Gesundheit zu erforschen - erst dann kann man beginnen Fragen wie in ihrer Habilitationsschrift „Was macht Frauen krank“ zu beantworten. Bestärkt worden darin ist sie sicher auch durch ihre Studien und Forschungsaufenthalte in den USA. Für die Praxis der Frauengesundheit war es immer bedeutsam auf diese Forschungsergebnisse hinweisen zu können.

Ihre Beiträge zur Sozial-Epidemiologie im Rahmen der Deutschen Herzkreislauf Präventionsstudie und zur gesundheitlichen Lage von Frauen in Deutschland kann man inzwischen wohl als Klassiker der deutschen Sozialepidemiologie bezeichnen. Aber auch ihre Hinweise auf die unterschiedliche Versorgung von Frauen und Männern bei gleichem Krankheitsbild z.B. Herzinfarkt - waren von entscheidender Bedeutung. Weibliche Patienten werden oft, auch aufgrund anderer Symptomatik, zu spät oder falsch diagnostiziert. Frauen sind immer noch in Studien zu Ursachen und Therapien von Krebserkrankungen stark unterrepräsentiert.

Salomon Neumann hat sein Wissen konsequent in die Politik eingebracht: Von 1858 bis 1905 gehörte er der Berliner Stadtverordnetenversammlung an. Ulrike Maschewsky-Schneider hat sich intensiv lokal in einer Vielzahl von Funktionen eingebracht: hier in Bremen vor 22 Jahren bei der Gründung des Frauengesundheitstreffpunkts Tenever und dem Verein Frauen und Gesundheit und Deutschlandweit im Beirat der Bundeskoordinierungsstelle Frauengesundheit; sie war hoch respektierte Sachverständige für wichtige Enquetekommissionen zur Frauenge- sundheit und frauengerechter Gesundheitsversorgung. Und auch in Berlin hat sie sich in Praxiseinrichtungen und Verbänden eingesetzt, so im Verein „Gesundheit Berlin e.V.“. Die Frauengesundheitszentren mussten sich stets am Schnittpunkt zur Politik bewähren als Vertreterinnen von Fraueninteressen in Gesundheit und Medizin und im Kampf um öffentliche Fördergelder.

Ulrike Maschewsky-Schneider hat integriert. Sie hilft mit ein bisher in Deutschland noch nicht existierendes interdisziplinäres Wissenschaftsfeld zu etablieren: die Gesundheitswissenschaften und habilitiert sich in der Sozialepidemiologie. Sie wird zur wichtigen Stimme für Public Health in Deutschland - im Vorstand dieser Gesellschaft und auch der deutschen Gesellschaft für Public Health. Sie hat entscheidend - in einer Vielzahl von Funktionen - daran mitgewirkt Public Health in Berlin mit am Leben zu erhalten.

Derzeit ist sie Sprecherin der Berlin School of Public Health an der Charité - wiederum eine Herausforderung in der Verwaltung mangelnder politischer und finanzieller Unterstützung. Bis heute ist es mir ein Rätsel warum es Deutschland als Land mit einer Tradition von Rudolf Virchow und Salomon Neumann es nicht schafft sich eine starke nationale School of Public Health zu geben, die über die Grenzen hinaus wirkt. Rudolf Virchow hat den Satz Neumanns weitergeführt: „Die Medizin ist ein soziale Wissenschaft und die Politik ist nichts weiter als Medizin im Großen“. Die Politik in Deutschland - alle Parteien - haben bis heute Public Health vernachlässigt. Eine Sisyphosarbeit für alle, die sich diesen Arbeitsfeldern verschrieben haben.

Ähnliches gilt weiter für die Frauengesundheit. Der WHO Bericht zur Frauengesundheit 2009 spricht eine deutliche Sprache: Societies and their health systems are failing women. Das bedeutet: auch noch heute - besonders mit einem globalen Blick - gibt es viel zu tun. Die Globalisierung hebt die Unterschiede nicht auf - sondern verteilt sie neu. Gender als Organisationsprinzip der Gesellschaft organisiert sich neu und mehr als uns lieb ist stehen Geschlecht und Gender - und damit die Konflikte um den Frauenkörper - im Zentrum des sozialen globalen Wandels.

Die Frauengesundheitsforschung und auch die Praxis steht vor neuen Herausforderungen - solche, die wir uns nicht hätten träumen lassen, bis hin zur Pornografisierung auch des Männerkörpers. Wenn der Körper ein Text ist „der uns viel darüber sagen kann wie Frauen (und auch Männer) in einer Gesellschaft gesehen werden und welchen Herausforderungen sie sich gegenüber sehen“ (Carolyn Knapp) dann sagen uns z. B. die Zunahme von Essstörungen bei den „global girls“ und die Zunahme von Depression bei jungen Männern nicht nur etwas über geschlechtliche Rollenidentität sondern auch über Körperbildstörungen durch die Sexualisierung und Pornografisierung der vermittelten globalen Bilder. Mädchen und Frauen sind heute für globale Industrien "the largest single product marketing opportunity in the world". Es beginnt mit dem Körper. Also es gilt weiterzumachen. Dies ist Aufgabe für mehr als eine Generation. Deshalb haben 2009 die Frauen von Our Bodies - Ourselves einen neuen Preis eingeführt: the Women's Health Heroes Award. Damit soll ein Zeichen gesetzt werden, das ich auch hier verwenden möchte: somit ich erkläre ich die Preisträgerinnen der 16 Frauengesundheitszentren für mich als Women's Health Heroes. Zum Schluss für Ulrike Maschewsky-Schneider ein Zitat der Frau, die wir alle immer wieder zitieren: Simone de Beauvoir. Aber nicht das übliche sondern ein Zitat über das Glück. Sie schreibt: „Das Glück ist eine Art Existenzberechtigung, die sich das Leben selbst verleiht“. Ich hoffe Ulrike Du warst glücklich. Für uns Frauen und für Public Health in Deutschland war es ein Glück dass Du bereit warst so viel von Dir zu geben.

Dankeschön. 